\title{
Effect of Probiotic Clostridium butyricum NCTC 7423 Supernatant on Biofilm Formation and Gene Expression of Bacteroides fragilis
}

\author{
Da-Seul Shin ${ }^{1}$, Ki-Jong Rhee ${ }^{2}$, and Yong-Bin Eom ${ }^{1,3 *}$ \\ ${ }^{1}$ Department of Medical Sciences, College of Medical Sciences, Soonchunhyang University, Asan 31538, Republic of Korea \\ ${ }^{2}$ Department of Biomedical Laboratory Science, College of Health Sciences, Yonsei University at Wonju, Wonju 26493, Republic of Korea \\ ${ }^{3}$ Department of Biomedical Laboratory Science, College of Medical Sciences, Soonchunhyang University, Asan 31538, Republic of Korea
}

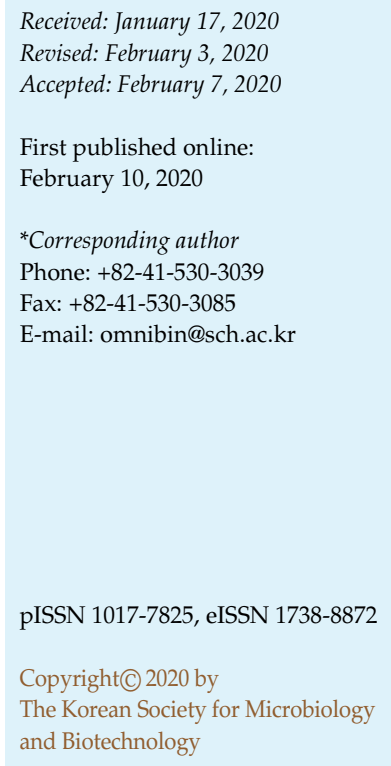

Enterotoxigenic Bacteroides fragilis (ETBF) is the main pathogen causing severe inflammatory diseases and colorectal cancer. Its biofilm plays a key role in the development of colorectal cancer. The objective of this study was to determine the antagonistic effects of cell-free supernatants (CFS) derived from Clostridium butyricum against the growth and biofilm of ETBF. Our data showed that C. butyricum CFS inhibited the growth of B. fragilis in planktonic culture. In addition, C. butyricum CFS exhibited an antibiofilm effect by inhibiting biofilm development, disassembling preformed biofilms and reducing the metabolic activity of cells in biofilms. Using confocal laser scanning microscopy, we found that C. butyricum CFS significantly suppressed the proteins and extracellular nucleic acids among the basic biofilm components. Furthermore, C. butyricum CFS significantly downregulated the expression of virulence- and efflux pump-related genes including ompA and bmeB3 in B. fragilis. Our findings suggest that $C$. butyricum can be used as biotherapeutic agent by inhibiting the growth and biofilm of ETBF.

Keywords: Bacteroides fragilis, Clostridium butyricum, probiotics, enterotoxigenic Bacteroides fragilis (ETBF), biofilm

\section{Introduction}

Bacteroides fragilis, an obligate anaerobe, constitutes $1 \%$ to $2 \%$ of the normal microorganisms in the intestines of a healthy human [1]. However, it is a major pathogen causing inflammatory bowel diseases (IBD) such as Crohn's disease and ulcerative colitis $[2,3]$. The virulence factors of $B$. fragilis such as capsular polysaccharide, outer membrane proteins and enterotoxin known as fragilysin (Bft) are closely associated with biofilm formation and pathogenicity of $B$. fragilis [4]. Above all, enterotoxigenic $B$. fragilis (ETBF) secreting Bft not only causes severe inflammatory diseases, but also contributes to the development of colorectal cancer (CRC) [5]. Recent studies have reported that more than $60 \%$ of the biofilms formed by ETBF secreting Bft are frequently found in patients with severe inflammatory diseases [6]. In addition, CRC development is affected by spatial arrangement of bacterial communities in a high-dimensional structure, indicating that biofilm shows pro-carcinogenic activity and is essential for CRC initiation [7]. Therefore, it is essential to identify an effective therapeutic alternative that prevents severe inflammatory diseases or CRC by inhibiting the growth and biofilm of ETBF secreting $\mathrm{Bft}$.

Bacteriotherapy using probiotics is a safe and promising approach [8]. A probiotic is defined as a live microorganism that provides health benefits when administered in appropriate amounts [9]. Probiotics have beneficial effects, such as maintaining intestinal homeostasis by regulating the host immune systems and suppressing the clustering of pathogens [10, 11]. Clostridium butyricum strains are probiotic bacteria that are used to prevent antibioticassociated diarrhea [12]. C. butyricum, an obligate anaerobe, is a common human and animal gut commensal bacterium and accounts for $10-20 \%$ of all human stool samples by microbial culture. They secrete an abundance of short- 
chain fatty acids (SCFAs), mainly butyrate and acetate, which promote the proliferation of enterocytes [13] and accelerate regulatory $\mathrm{T}$ cell generation [14]. C. butyricum also possesses antifungal and antibacterial activities against Candida albicans, Clostridium difficile, enterotoxigenic E. coli (ETEC), Vibrio spp. and Helicobacter pylori [15-17]. Further, a recent study by Hayashi et al. revealed that $C$. butyricum represses acute experimental colitis in mice by stimulating intestinal interleukin-10 (IL-10)-producing macrophages [18].

Although studies have actively investigated probiotics, few studies have analyzed the inhibitory effects of $C$. butyricum on intestinal biofilm formation by pathogens. Especially, the effect of $C$. butyricum against the growth and biofilm of ETBF secreting Bft has yet to be reported. In order to investigate the effect and the possible application of metabolites formed by C. butyricum, this study evaluated the antagonistic activity of cell-free supernatant (CFS) extracted from C. butyricum against the growth, biofilm formation and gene expression of $B$. fragilis. Our work widens our intellectual horizon on the potential antibacterial and antibiofilm activities of CFS derived from C. butyricum in addition to its role as biotherapeutic agent against ETBF secreting Bft.

\section{Materials and Methods}

\section{Organisms, Media and Growth Conditions}

The wild-type enterotoxigenic Bacteroides fragilis 86-5443-2-2 (WT-ETBF; bft-2), a wild-type non-enterotoxigenic Bacteroides fragilis NCTC 9343 (WT-NTBF), and a recombinant strain transformed via insertion of bft-2 gene into WT-NTBF (rETBF; bft-2) and C. butyricum NCTC 7423 (ATCC 19398), a nontoxigenic strain, were used in this study [19]. B. fragilis strains and C. butyricum NCTC 7423 were cultured in brain heart infusion (BHI; Difco, BectonDickinson and Company, USA) supplemented with $1 \%$ glucose, $0.1 \%$ hemin, $0.5 \%$ yeast extract, and $0.05 \%$ L-cystine (BHIS) and reinforced clostridial medium (RCM; Difco, Becton-Dickinson and Company), respectively, at $37^{\circ} \mathrm{C}$ under anaerobic conditions [20]. All stock cultures were stored in broth with $20 \%$ glycerol at $-80^{\circ} \mathrm{C}$ until testing.

\section{Preparation of $C$. butyricum Cell-Free Supernatant}

The C. butyricum cell-free supernatant (CFS) was prepared as described previously [21]. In brief, C. butyricum NCTC 7423 was incubated with the reinforced clostridial medium at $37^{\circ} \mathrm{C}$ for $24 \mathrm{~h}$ under anaerobic conditions. The culture was centrifuged at 3,000 rpm for $15 \mathrm{~min}$ and the CFS was filtered with $0.2 \mu \mathrm{m}$ pore size syringe filter (Advantec, Japan). To determine whether the inhibitory activity may beaffected by the bacteriocin-like inhibitory substances, the CFS was adjusted to $\mathrm{pH} 6.5$ using $1 \mathrm{~N}$
$\mathrm{NaOH}$ to exclude the effect of organic acid [22].

\section{C. butyricum CFS Susceptibility Testing on B. fragilis Planktonic Cells}

To determine the antibacterial activity of CFS derived from $C$. butyricum, the broth microdilution method was used according to the Clinical and Laboratory Standards Institute guidelines [23], with a few modifications. Briefly, overnight cultures of $B$. fragilis strains were grown in BHIS broth. The bacterial suspension was adjusted to the final concentration of $1 \times 10^{6} \mathrm{CFU} / \mathrm{ml}$ and inoculated into a 96-well microtiter plate (BD Falcon, USA) along with the C. butyricum CFS or neutralized CFS to a total volume of $200 \mu$ l. The C. butyricum CFS or neutralized CFS was serially diluted two-fold in BHIS broth. Overnight-cultured B. fragilis strains were set as control. Following inoculation, the 96-well microtiter plate was incubated anaerobically at $37^{\circ} \mathrm{C}$ for $24 \mathrm{~h}$. At the end of the incubation, the growth of planktonic cells was assessed at $600 \mathrm{~nm}\left(\mathrm{~A}_{600}\right)$ wavelength using a Multiskan GO plate reader (Thermo Fisher Scientific, USA).

\section{Effect of C. butyricum CFS on Biofilm Formation and Preformed Biofilms of $B$. fragilis}

The biofilm formation assay was performed in a 96-well microtiter plate as described previously [24, 25], with some modifications. Overnight-cultured B. fragilis suspensions were diluted in BHIS broth to a final density of $1 \times 10^{6} \mathrm{CFU} / \mathrm{ml}$ and dispensed into a 96-well microtiter plate. The 2-fold serial dilutions of C. butyricum CFS in BHIS broth were added to wells of a 96-well microtiter plate and incubated at $37^{\circ} \mathrm{C}$ for $24 \mathrm{~h}$ under anaerobic conditions.

To establish the preformed biofilms, a reference to $B$. fragilis were prepared in BHIS broth at a density of $1 \times 10^{6} \mathrm{CFU} / \mathrm{ml}$, added to a 96-well microtiter plate and incubated anaerobically at $37^{\circ} \mathrm{C}$ for $24 \mathrm{~h}$. At the end of the incubation, the preformed biofilms were rinsed with $0.01 \mathrm{M}$ phosphate-buffered saline (PBS; pH 7.4) to remove non-adherent cells. C. butyricum CFS was diluted two-fold in fresh BHIS broth and inoculated into the preformed biofilms. A 96-well microtiter plate was incubated at $37^{\circ} \mathrm{C}$ for an additional $24 \mathrm{~h}$ under anaerobic conditions. The control was set as mentioned above. The inhibitory effect of C. butyricum CFS on biofilm formation and preformed biofilms of $B$. fragilis was quantitatively evaluated using the crystal violet assay as described below.

\section{Biofilm Quantitation Via Crystal Violet Assay}

The crystal violet assay was performed to evaluate the biofilm biomass quantitatively as described previously [25, 26], with minor modifications. After the biofilm formation, the medium was aspirated and the biofilm was washed with PBS to remove unattached cells from wells. The 96-well microtiter plate was dried at $60^{\circ} \mathrm{C}$ for $1 \mathrm{~h}$. The remaining biofilms were stained with $1 \%$ crystal violet for $5 \mathrm{~min}$ and rinsed with sterile distilled water to remove the dye. After drying at $60^{\circ} \mathrm{C}$ for $1 \mathrm{~h}$, the crystal violet 
was extracted from the stained biofilms using 33\% (v/v) acetic acid. The biofilm biomass was quantitatively determined by measuring absorbance at $570 \mathrm{~nm}\left(\mathrm{~A}_{570}\right)$.

\section{Biofilm Metabolic Activity - XTT Reduction Assay}

The metabolic activity of $B$. fragilis biofilms was analyzed using the XTT [2,3-bis(2-methoxy-4-nitro-5-sulfophenyl)-2H-tetrazolium5-carboxanilide] reduction assay as described previously [27], with slight modifications. Briefly, the 96-well microtiter plate was used to establish the biofilms of three $B$. fragilis strains as described above. After the preformed biofilms were washed with PBS, a fresh BHIS broth containing the 2-fold serial dilutions of $C$. butyricum CFS was dispensed into preformed biofilms and incubated at $37^{\circ} \mathrm{C}$ for $24 \mathrm{~h}$ in anaerobic environments. Subsequently, the preformed biofilms were washed with PBS. The metabolic activity of $B$. fragilis biofilms was determined using the XTT cell proliferation assay kit (ATCC, USA) in accordance with the manufacturer's instructions. Prior to the experiment, the XTT reagent was mixed with the activation reagent at a ratio of 50:1 (v/v), and $50 \mu \mathrm{l}$ of the XTT/activation was dispensed into the preformed biofilms and incubated at $37^{\circ} \mathrm{C}$ in the dark for $3 \mathrm{~h}$. To analyze the metabolic activity of $B$. fragilis biofilms, the specific absorbances were calculated at a test wavelength of $475 \mathrm{~nm}\left(\mathrm{~A}_{475}\right)$ and a reference wavelength of $650 \mathrm{~nm}\left(\mathrm{~A}_{650}\right)$ [28]. According to the XTT cell proliferation assay protocol, the specific absorbance of the sample is expressed mathematically as follows; Specific absorbance $=\mathrm{A}_{475}$ (Test) $-\mathrm{A}_{475}$ (Blank) $-\mathrm{A}_{650}$ (Test).

\section{Confocal Laser Scanning Microscopy}

Confocal laser scanning microscopy (CLSM) was conducted using biofilms of three $B$. fragilis strains developed on a tissue culture-treated 24-well glass bottom imaging plate (Eppendorf AG, Germany, Cat. no.: 0030741021) as reported previously [29], with some modifications. B. fragilis strains in BHIS were dispensed into the wells at a concentration of $1 \times 10^{6} \mathrm{CFU} / \mathrm{ml}$ with 2-fold serial dilutions of C. butyricum CFS and incubated at $37^{\circ} \mathrm{C}$ for $24 \mathrm{~h}$ under anaerobic conditions. Overnight-cultured B. fragilis strains were employed as control. Following incubation, biofilms formed on the well were washed with PBS and fixed with 3.7\% $(\mathrm{v} / \mathrm{v})$ formaldehyde for $1 \mathrm{~h}$. The amino groups in the $B$. fragilis biofilms were visualized by staining with fluorescein isothiocyanate isomer I (FITC, $10 \mu \mathrm{g} / \mu \mathrm{l}$; Sigma-Aldrich, Germany) for $1 \mathrm{~h}$. Carbohydrates of biofilms were stained with Concanavalin A-Alexa Fluor 594 conjugate (Con A, $0.1 \mu \mathrm{g} / \mu \mathrm{l}$; C-11253, Molecular Probes, USA) for $30 \mathrm{~min}$. To monitor the extracellular nucleic acids, biofilms were stained with 4, 6-diamidino-2-phenylindoldihydrochloride (DAPI, $1 \mathrm{mg} / \mathrm{l}$; Molecular Probes) for $45 \mathrm{~min}$. After each step, the stained biofilms were washed with PBS to remove unbound staining solution. All steps were conducted in a dark room. The biofilms of $B$. fragilis strains were visualized at excitation wavelengths of 495,590 , and $358 \mathrm{~nm}$ for FITC, Con A and DAPI, respectively, using Zeiss LSM-710 confocal laser microscope (Carl Zeiss, USA) and were imaged using ZEN software (Carl Zeiss).
Table 1. Primer sequences used for qRT-PCR.

\begin{tabular}{clc}
\hline Name & \multicolumn{1}{c}{ Sequence $\left(5^{\prime} \rightarrow 3^{\prime}\right)$} & Reference \\
\hline 16S rRNA & $\begin{array}{l}\text { Forward AGTAGAGGTGGGCGGAATTC } \\
\text { Reverse GTGTCAGTTGCAGTCCAGTG }\end{array}$ & {$[30]$} \\
& Forward GGATATGACGGTGTTGCCAG & {$[31]$} \\
& Reverse TAGCAGCAGCCATGTCATTC & \\
bmeB3 & Forward GTACCGGAAGTTCAAGGTGT & {$[32]$} \\
& Reverse GAGCAGCCTCGATATTCTGT & \\
\hline
\end{tabular}

\section{RNA Extraction and Quantitative Real-Time Polymerase Chain Reaction (qRT-PCR)}

To extract the total RNA of B. fragilis strains, $1 \times 10^{6} \mathrm{CFU} / \mathrm{ml}$ of B. fragilis in BHIS was inoculated with C. butyricum CFS serially diluted 2-fold and incubated at $37^{\circ} \mathrm{C}$ for $24 \mathrm{~h}$ in anaerobic environments. At the end of incubation, the cells were collected by centrifuging each bacterial suspension at 25,000 $\times g$ for $1 \mathrm{~min}$ at $4^{\circ} \mathrm{C}$. The total RNA of $B$. fragilis strains was extracted and purified through NucleoSpin RNA mini Kit (Macherey-Nagel, Germany) in compliance with the manufacturer's protocol. The concentration and purity of extracted RNA was assessed using BioDrop $\mu$ LITE (BioDrop Ltd., UK), and $1 \mu \mathrm{g}$ of template was reverse-transcribed into cDNA in a $20 \mu \mathrm{l}$ reaction volume, using ReverTra Ace qPCR RT Master Mix with gDNA Remover (TOYOBO, Japan). The qRTPCR was performed to investigate the relative gene expression of B. fragilis outer membrane protein (ompA) associated with virulence factor and RND-type efflux pump-related bmeB3. Power SYBR Green PCR Master Mix (Applied Biosystems, USA) was employed to analyze the PCR amplification products. The qRTPCR was conducted using a StepOnePlus Real-Time PCR System (Applied Biosystems). Primer sequences used in qRT-PCR are shown in Table 1 [30-32]. 16S rRNA was used as a housekeeping gene. The thermal cycling conditions of qRT-PCR were as follows: an initial denaturation at $95^{\circ} \mathrm{C}(10 \mathrm{~min})$, followed by 40 cycles of denaturation at $95^{\circ} \mathrm{C}(15 \mathrm{sec})$, annealing at $58^{\circ} \mathrm{C}(1 \mathrm{~min})$, and extension at $72^{\circ} \mathrm{C}(20 \mathrm{sec})$. The annealing stage was set at $57^{\circ} \mathrm{C}$ $(30 \mathrm{sec})$ for the $o m p A$ gene and $55^{\circ} \mathrm{C}(1 \mathrm{~min})$ for the $b m e B 3$ gene. The relative gene expression of target genes was normalized to $16 S$ rRNA gene and evaluated using the formula of $2^{-\Delta \Lambda C T}$.

\section{Statistical Analysis}

All data are representative of three independent experiments, and the data were indicated as means \pm standard deviations (SD). The results were analyzed via one-way analysis of variance (ANOVA) followed by Dunnett's test to evaluate the significant differences between the treated groups and the control group. The results of qRT-PCR were analyzed using the Student's $t$-test. All statistical analyses were conducted using the GraphPad Prism version 5 (GraphPad Software, USA). Statistical significance was considered at ${ }^{*} p<0.05,{ }^{* *} p<0.01$ and ${ }^{* *} p<0.001$. 

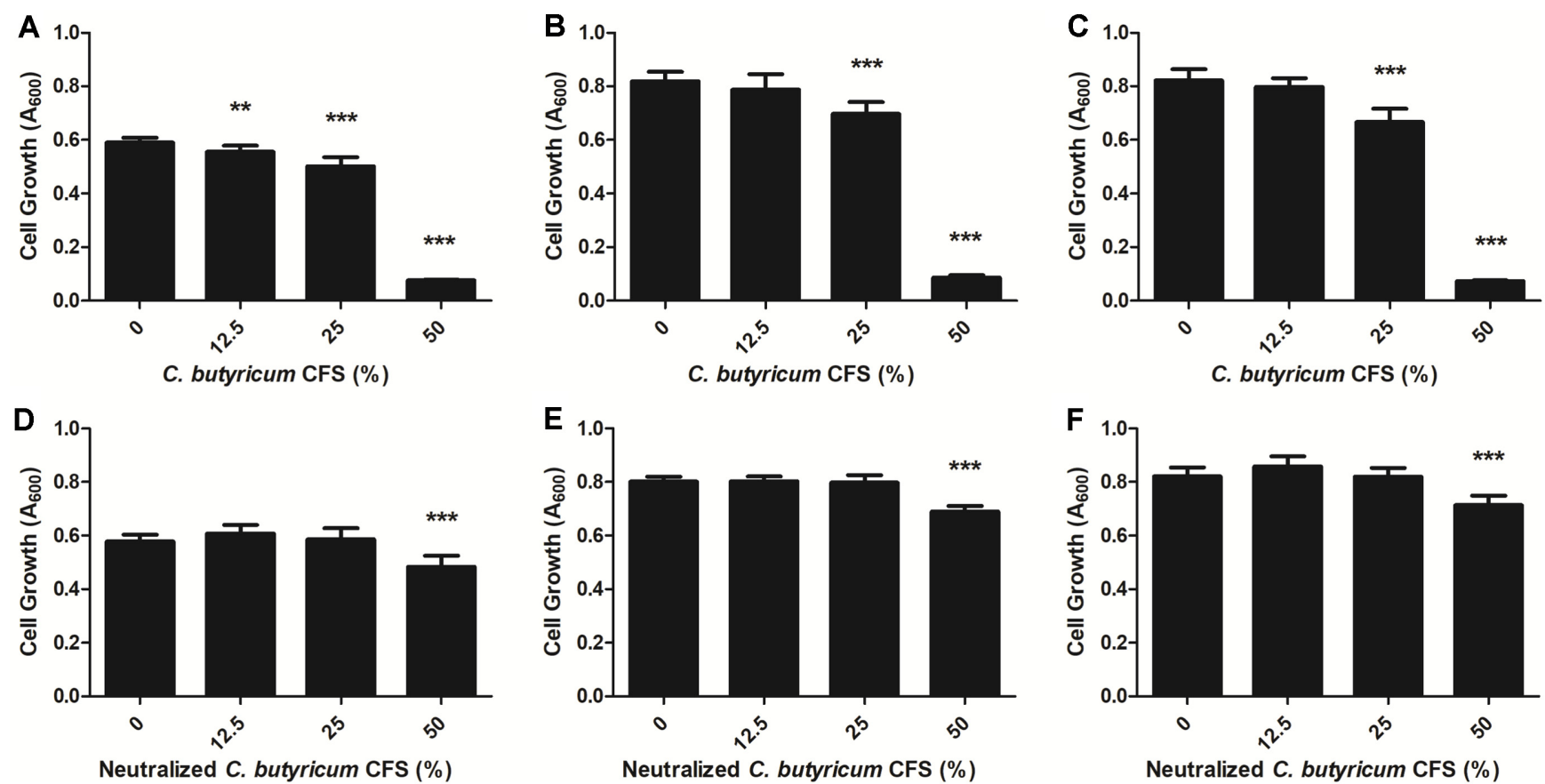

Fig. 1. Antibacterial activity of $C$. butyricum CFS against the growth of $B$. fragilis planktonic cells.

WT-ETBF (bft-2) (A, D), rETBF (bft-2) (B, E) and WT-NTBF (C, F) were incubated in the presence of C. butyricum CFS (A-C) or neutralized C. butyricum CFS (D-F) at $37^{\circ} \mathrm{C}$ for $24 \mathrm{~h}$ under anaerobic conditions. The growth of $B$. fragilis planktonic cells was analyzed at $\mathrm{A}_{600}$ using microplate spectrophotometers. The results are expressed as means \pm standard deviations (SD). ${ }^{* *}$ and ${ }^{* * *}$ describe significant differences at $p<0.01$ and $p<$ 0.001 , respectively.

\section{Results}

\section{Antibacterial Activity of C. butyricum CFS on B. fragilis} Planktonic Cells

The antibacterial activity of C. butyricum CFS against $B$. fragilis strains was determined by measuring growth inhibition. A 50\% C. butyricum CFS completely inhibited the growth of WT-ETBF ( $b f t-2)$, rETBF ( $b f t-2)$ and WT-NTBF by $99.48 \%, 98.38 \%$ and $100 \%$, respectively (Figs. 1A-1C). A $25 \%$ C. butyricum CFS has a lower potential than $50 \%$, but it significantly reduced the growth of $B$. fragilis strains. However, the growth of rETBF (bft-2) and WT-NTBF was not inhibited by $12.5 \%$ C. butyricum CFS. C. butyricum suppresses the growth of other microorganisms by producing a bacteriocin-like inhibitory compound [33]. In addition, neutralized probiotic CFS shows a partial similar effect to bacteriocin [21]. Therefore, to evaluate the effect of neutralized C. butyricum on the antibacterial activity, the $C$. butyricum CFS was adjusted to $\mathrm{pH} 6.5$ to exclude organic acids in CFS. With the treatment of $50 \%$ C. butyricum CFS, the growth inhibition of three $B$. fragilis strains showed a statistically significant difference (Figs. 1D-1F). However, treatment with the neutralized C. butyricum CFS yielded less antibacterial activity than that of C. butyricum CFS against $B$. fragilis (Fig. 1), suggesting that the bacteriocin in C. butyricum CFS does not affect its growth.

\section{Inhibitory Effect of C. butyricum CFS on B. fragilis Biofilm Formation}

To determine the inhibitory effect of $C$. butyricum CFS on the biofilm formation of three $B$. fragilis strains, the biofilm was quantified by staining with crystal violet and measuring its absorbance at $570 \mathrm{~nm}\left(\mathrm{~A}_{570}\right)$ wavelength. The biofilm formation of all strains used in this study was reduced by the C. butyricum CFS in a dose-dependent manner. More specifically, the biofilm formation of WT-ETBF (bft-2) was inhibited by $46.46 \%, 55.51 \%$ and $95.89 \%$ in the presence of $12.5 \%, 25 \%$, and $50 \%$ C. butyricum CFS, respectively (Fig. 2A). The rETBF (bft-2) was also suppressed by $32.86 \%$, $35.29 \%$, and $93.48 \%$ (Fig. 2B) and WT-NTBF was inhibited by $30.16 \%, 48.61 \%$, and $90.5 \%$ (Fig. 2C) following exposure to $12.5,25$, and $50 \%$ C. butyricum CFS, respectively. The results showed that the biofilm formation of all $B$. fragilis strains was inhibited by more than $90 \%$ by treatment with $50 \%$ C. butyricum CFS. 

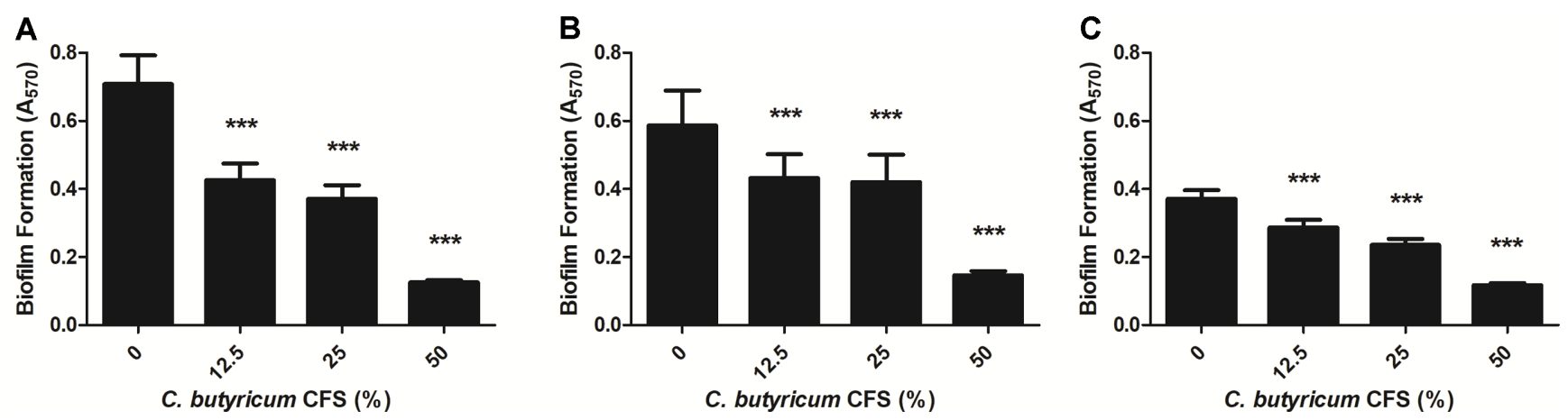

Fig. 2. Inhibitory effect of C. butyricum CFS on biofilm formation of B. fragilis.

WT-ETBF (bft-2) (A), rETBF (bft-2) (B) and WT-NTBF (C) were incubated with C. butyricum CFS at $37^{\circ} \mathrm{C}$ for $24 \mathrm{~h}$ under anaerobic conditions. Biofilms of $B$. fragilis strains were stained with $1 \%$ crystal violet and evaluated by measuring the absorbance at $A_{570}$. The data are presented as means \pm standard deviations (SD). ${ }^{* * *} p<0.001$ means statistical significance compared with the control group.

Effect of C. butyricum CFS on Preformed Biofilms of $B$. fragilis

As shown above, the C. butyricum CFS not only inhibited the biofilm formation of $B$. fragilis strains, but also potentially eliminated such preformed biofilms. In the presence of 12.5 to $25 \%$ C. butyricum CFS, the preformed biofilms by WT-ETBF (bft-2), rETBF (bft-2), and WT-NTBF were eradicated by 9.38 to $26.11 \%, 54.22$ to $72.05 \%$, and 49.28 to $71.9 \%$, respectively (Fig. 3). Although the preformed biofilms of WT-ETBF ( $b f t-2)$ were less affected than rETBF (bft-2) and WT-NTBF upon treatment with 12.5 to $25 \%$ C. butyricum CFS, the preformed biofilms of WT-ETBF (bft-2) were significantly eliminated by $93.24 \%$ upon treatment with $50 \%$ C. butyricum CFS (Fig. 3A). The rETBF (bft-2) was also eliminated by $90.19 \%$ at the same concentration (Fig. 3B). However, only $85 \%$ of the WT-NTBF preformed biofilms were eliminated by treatment with $50 \%$ C. butyricum CFS (Fig. 3C).

Inhibitory Effect of C. butyricum CFS on the Metabolic Activity of Established B. fragilis Biofilms: XTT Reduction Assay

A colorimetric XTT reduction assay was performed to determine the viability of $B$. fragilis cells within the biofilms in the presence of $C$. butyricum CFS. The specific absorbance was calculated using an XTT reduction assay. Even though the viability of WT-ETBF (bft-2) biofilm was less than $78.65 \%$ compared with other strains upon treatment with $25 \%$ C. butyricum CFS, the viability of all B. fragilis strains used in this study declined by more than $90 \%$ with $50 \%$ C. butyricum CFS (Fig. 4). As shown in Fig. 4, the metabolic activities of all strains were significantly reduced by
A

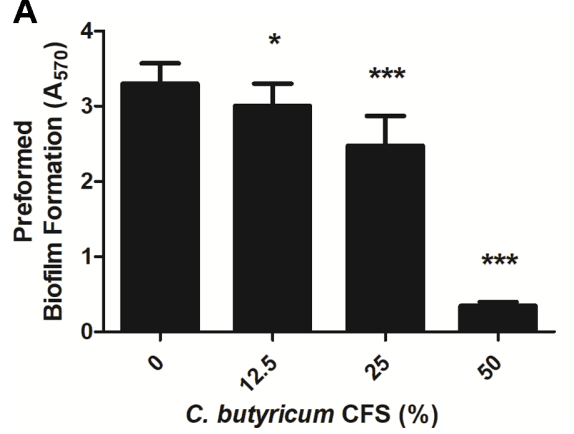

B

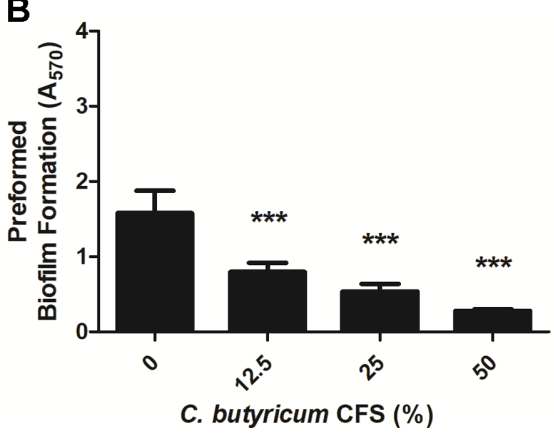

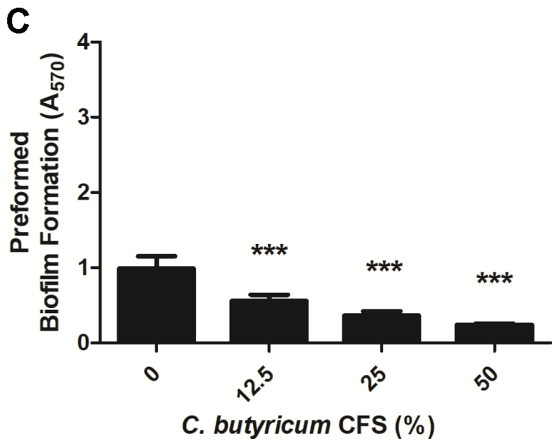

Fig. 3. Eradicative effect of C. butyricum CFS on preformed biofilms of B. fragilis.

The preformed biofilms of WT-ETBF (bft-2) (A), rETBF (bft-2) (B), and WT-NTBF (C) were incubated anaerobically at $37^{\circ} \mathrm{C}$ for $24 \mathrm{~h}$ in the presence of C. butyricum CFS. Biofilms of B. fragilis strains were stained with $1 \%$ crystal violet and determined by measuring at $\mathrm{A}_{570}$. The data are presented as means \pm standard deviations (SD). ${ }^{*} p<0.05$ and ${ }^{* * *} p<0.001$ indicate statistical significance compared with the control group. 

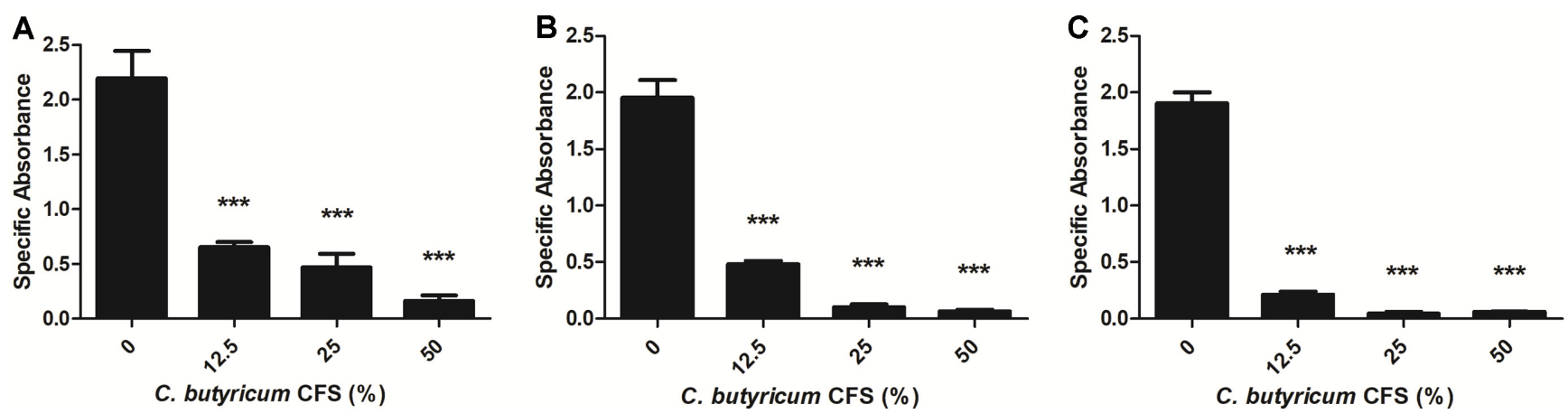

Fig. 4. Inhibitory effect of $C$. butyricum CFS on the metabolic activity of established biofilms.

The established biofilms of WT-ETBF (bft-2) (A), rETBF (bft-2) (B), and WT-NTBF (C) were incubated anaerobically at $37^{\circ} \mathrm{C}$ for $24 \mathrm{~h}$, along with $C$. butyricum CFS. The metabolic activity of biofilm cells was assessed using XTT reduction assay. Specific absorbance was calculated as $\mathrm{A}_{475}$ (Test) $\mathrm{A}_{475}$ (Blank) $-\mathrm{A}_{650}$ (Test). The results are presented as means \pm standard deviations (SD). Asterisks ${ }^{(* * *)}$ signify $p<0.05$ compared with the control group.

treatment with the C. butyricum CFS in a dose-dependent manner. The results suggest that treatment with $C$. butyricum CFS suppresses the viability of $B$. fragilis cells within the biofilms as well as inhibits and eliminates $B$. fragilis biofilms.

\section{Confocal Laser Scanning Microscopy}

The extracellular polymeric substances (EPS) of biofilm matrix are mainly composed of proteins, carbohydrates and extracellular DNAs [34]. To visualize the inhibitory effect of C. butyricum CSF on the biofilms of $B$. fragilis strains, CLSM was performed by staining biofilms with fluorescent dyes. In the absence of C. butyricum CFS, the biofilms of WT-ETBF (bft-2), rETBF (bft-2) and WT-NTBF showed a thick and compact extracellular matrix and robust bacterial growth, as shown in Figs. 5A, 5C, and 5E, respectively. In addition, bacterial cell aggregation was clearly observed in the biofilms of all strains. By contrast, biofilms of all strains treated with $25 \%$ C. butyricum CFS were dispersed and disassembled, and bacterial cells were scattered due to lack of structural components compared with the untreated group (Figs. 5B, 5D, and 5F). Proteins and extracellular nucleic acids in the biofilm were remarkably reduced after treatment with $C$. butyricum CFS. These results show that $C$. butyricum $C F S$ alters the architecture of extracellular matrix and reduces the cell density, thickness, and biomass of biofilms.

Effect of $C$. butyricum CFS on the Expression of Virulenceand Efflux Pump-Related Genes in B. fragilis

To obtain further insight into the molecular mechanism of C. butyricum CFS underlying the inhibition of B. fragilis biofilms, qRT-PCR analysis was performed. The expression of ompA and bmeB3 genes in WT-ETBF (bft-2) and rETBF (bft-2) was considerably down-regulated by C. butyricum CFS in a concentration-dependent manner, whereas that of WT-NTBF was not affected (Fig. 6). Thus, exposure to $6.25 \%, 12.5 \%$, and $25 \%$ concentrations of C. butyricum CFS downregulated the expression of ompA gene in WT-ETBF (bft-2) by 4.22-fold, 6.11-fold, and 7.57-fold, respectively (Fig. 6A), and reduced the expression of ompA gene in rETBF ( $b f t-2$ ) by 1.48-fold, 2.73-fold, and 3.17-fold, respectively (Fig. 6B). At similar concentrations, the expression of bmeB3 gene in WT-ETBF (bft-2) was dramatically suppressed by 101.13-fold, 131.6-fold, and 136.82-fold, respectively (Fig. 6D), and the expression of bmeB3 gene in rETBF (bft-2) was downregulated by 1.37 -fold, 1.63-fold, and 1.87-fold, respectively (Fig. 6E). However, the levels of ompA and bmeB3 gene in WT-NTBF were almost unaffected by the $C$. butyricum CFS, and the differences were not statistically significant (Figs. 6C and 6F).

\section{Discussion}

Biofilms of ETBF carrying the bft gene are involved in the development of severe inflammatory diseases and CRC [7, 35]. Also, biofilm can lead to side effects such as selection of antibiotic-resistant bacteria and suppression of host immune system. For this reason, probiotics represent an alternative approach to preventing growth and biofilm of B. fragilis carrying the bft gene. C. butyricum, a probiotic, can selectively kill pathogens without affecting the normal intestinal flora [24] -and possess anti-diabetic [36], antibacterial, antifungal [17] and anticancer effects [37]. However, the inhibition of $B$. fragilis by $C$. butyricum has yet 


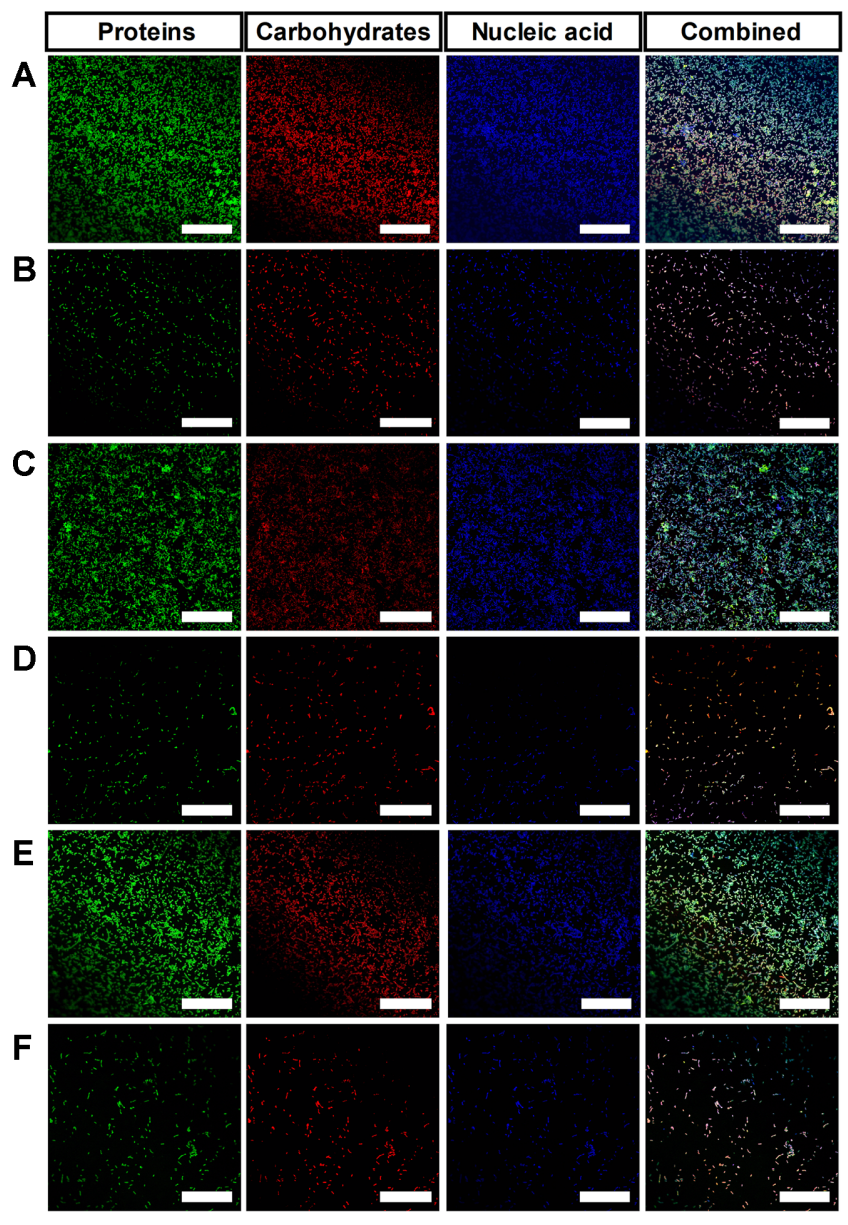

Fig. 5. Confocal laser scanning microscopy (CLSM) images of B. fragilis biofilms.

Proteins, carbohydrates, and nucleic acids occurring in B. fragilis biofilms were stained with FITC (Green), Con A (Red), and DAPI (Blue), respectively. WT-ETBF (bft-2) control (A) and after treatment with C. butyricum CFS (B). rETBF (bft-2) control (C) and after treatment with C. butyricum CFS (D). WT-NTBF control group (E) and after treatment with C. butyricum CFS (F). Biofilms were examined at $40 \times$ magnification. The scale bar indicates $50 \mu \mathrm{m}$.

to be reported. Therefore, our findings show that CFS extracted from C. butyricum significantly inhibits the growth and biofilm formation by ETBF carrying the bft gene.

The Bft toxin is a major virulence factor of $B$. fragilis. According to a study conducted by Pierce and Bernstein [38], ETBF carrying the bft gene was formed via mutations in non-toxigenic (NTBF) strain through evolutionary changes. Therefore, this study was conducted using WTETBF (bft-2), rETBF (bft-2), and WT-NTBF to confirm that CFS extracted from C. butyricum was strain-specific.

Meanwhile, C. butyricum produces bacteriocin-like inhibitory substances as well as organic acids, mainly butyrate. Bacteriocins are proteinaceous toxins produced by bacteria to inhibit the growth of similar or closely related bacterial strains [39]. Bacteriocin isolated from C. butyricum exhibited antimicrobial effects on diverse bacteria, but no effects on gram-negative bacteria [33]. Consistent with previous studies, our results also show that neutralized CFS, which has the same effect as bacteriocin, do not strongly inhibit the growth of B. fragilis.

HPLC analysis of Isono's groups revealed that $C$. butyricum CFS contained $13.6 \mathrm{mmol}$ butyrate, $8.3 \mathrm{mmol}$ acetate, and $4.3 \mathrm{mmol}$ formate [40]. Also, other studies confirmed that the amount of butyric acid among SCFAs increased most significantly when C. butyricum was inoculated on the in vivo models that caused various diseases [41, 42]. Butyrate, which accounts for most of the short-chain fatty acids (SCFAs) produced by C. butyricum, is soluble in water and has amphipathic actions [43]. Since $97 \%$ of the biofilm is composed of water, it is indicated that CFS (mostly butyrate) derived from C. butyricum can effectively pass throughout the biofilm [44, 45]. In addition, many studies have determined that butyric acid has an inhibitory effect on the growth and biofilm of various microorganisms such as Trichosporon spp., Vibrio spp., Clostridium difficile [45-47]. For these reasons, we expect that the action of the butyric acid is involved in the inhibitory effect of C. butyricum CFS against $B$. fragilis biofilm.

A previous study demonstrated that ETBF carrying the bft gene formed a large amount of biofilm compared with the non-toxigenic (NTBF) strain [38]. Similarly, our results also show that WT-ETBF (bft-2) and rETBF (bft-2) with the $b f t$ gene form more biofilms than the WT-NTBF without the bft gene. Nevertheless, our data showed that the C. butyricum CFS more effectively inhibited and eradicated biofilms of WT-ETBF (bft-2) and rETBF (bft-2) with the bft gene than WT-NTBF without the bft gene.

Furthermore, this study analyzed the expression levels of omp $A$ and bmeB3 genes according to the concentration of $C$. butyricum CFS by qRT-PCR. More specifically, the ompA, the most abundant outer membrane protein in B. fragilis, plays a structural role in capsule formation [48]. Lilian et al. have shown a positive correlation between increased adhesion and ompA gene expression in B. fragilis, suggesting that $о т p A$ gene was associated with adherence to human intestinal epithelial cells [48]. In addition, the ompA protein is involved in maintaining cell structure in the biofilms of B. fragilis [31]. Based on previous studies, it can be assumed that ompA gene plays a major role in the adhesion and biofilm formation of $B$. fragilis. Moreover, $B$. fragilis contains 

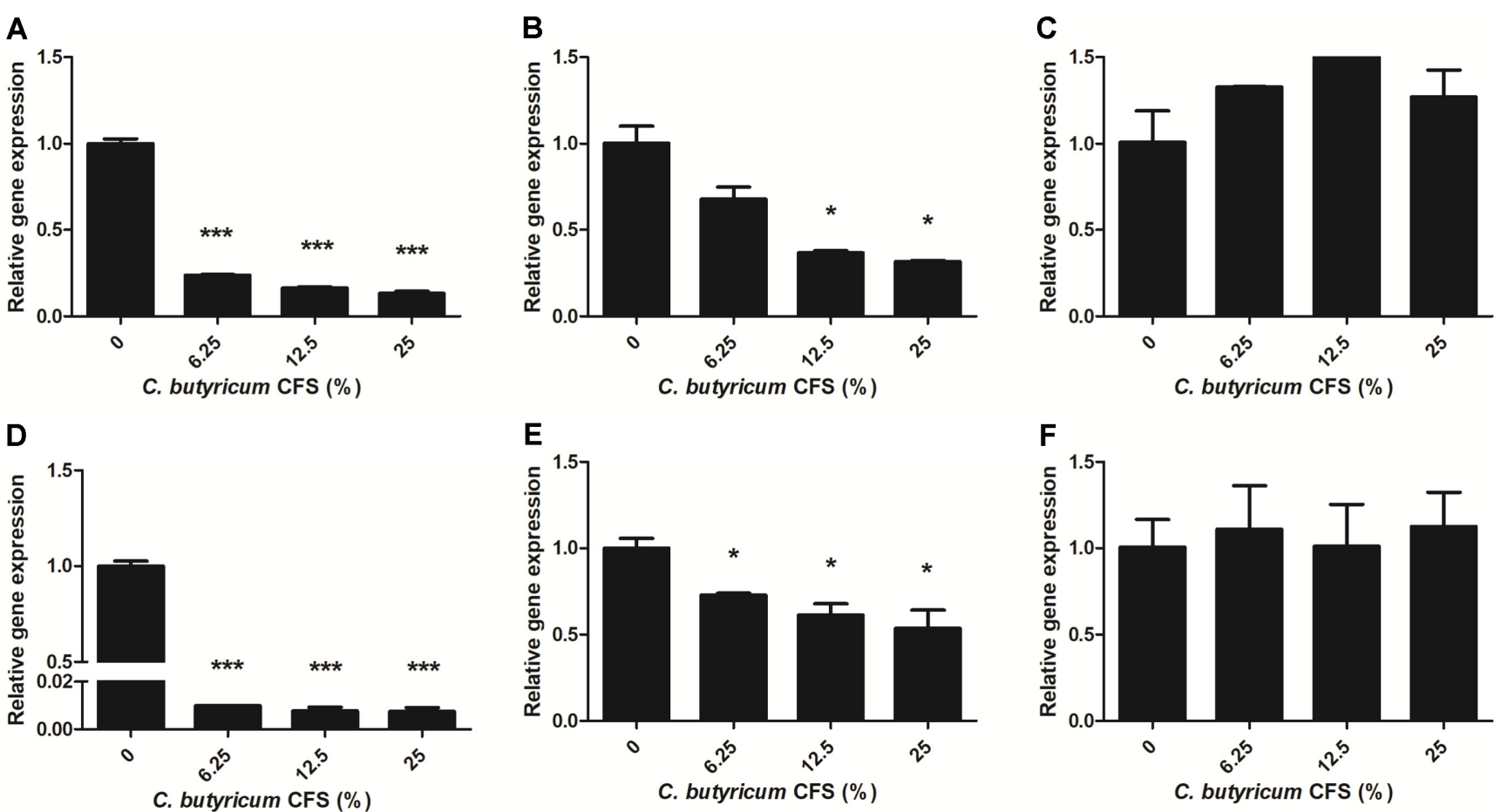

Fig. 6. qRT-PCR analysis of $B$. fragilis outer membrane protein gene ompA and RND-type efflux pump-related gene $b m e B 3$. ompA gene expression of WT-ETBF (bft-2) (A), rETBF (bft-2) (B), and WT-NTBF (C). bmeB3 gene expression of WT-ETBF (bft-2) (D), rETBF (bft-2) (E) and WT-NTBF (F). The results are presented as fold changes relative to the control group. Student's $t$-test was conducted to analyze the gene expression between treated and control groups. The results are presented as means \pm standard deviations (SD). ${ }^{*} p<0.05$ and ${ }^{* * *} p<0.001$ indicate statistical significance compared with the control group.

putative $\operatorname{luxR}$ orthologues that control biofilm formation, bmeB efflux pump expression, and susceptibility to antibiotics [49]. Also, the RND-type drug efflux pumps from gram-negative bacteria are related to virulence and biofilm formation [50]. This finding suggests that expression of bmeB gene related to RND-type efflux pump is associated with $B$. fragilis biofilm formation. Our study shows that the C. butyricum CFS inhibits biofilm formation of WT-ETBF and rETBF strains carrying the toxigenic bft-2 gene by reducing the expression of virulence- and efflux pumprelated genes (ompA and bmeB3) in B. fragilis. Our results indicated that $C$. butyricum CFS suppressed ompA and bmeB3 genes only for $B$. fragilis strains with the bft gene.

Thus, our findings showed that CFS derived from $C$. butyricum exhibits antibacterial and antibiofilm activities against ETBF carrying the toxigenic $b f t-2$ gene by regulating the virulence- and efflux pump-related genes (ompA and bmeB3). This study demonstrates the effectiveness of CFS obtained from C. butyricum against biofilm formation and provides scientific evidence for the development of new antibacterial and antibiofilm agents. Furthermore, these results suggest the potential of $C$. butyricum CFS as biotherapeutic agent to prevent and treat IBD and/or CRC caused by ETBF. Since this study does not analyze the chemical composition of C. butyricum CFS, further study needs to thoroughly investigate the effects of the one major component in C. butyricum CFS against the growth and biofilm of ETBF. Although further studies are needed to determine its clinical application, the results suggest that $C$. butyricum acts as an effective probiotic against ETBF secreting Bft by reducing growth and biofilm formation.

\section{Acknowledgments}

This work was supported by Soonchunhyang University research fund and a grant (NRF-2017R1D1A1B03032960) of the Basic Science Research Program through the National Research Foundation of Korea (NRF) funded by the Ministry of Education.

\section{Conflict of Interest}

The authors have no financial conflicts of interest to declare. 


\section{References}

1. Prindiville TP, Sheikh RA, Cohen SH, Tang YJ, Cantrell MC, Silva J, Jr. 2000. Bacteroides fragilis enterotoxin gene sequences in patients with inflammatory bowel disease. Emerg. Infect. Dis. 6: 171-174.

2. Wexler HM. 2007. Bacteroides: the good, the bad, and the nitty-gritty. Clin. Microbiol. Rev. 20: 593-621.

3. Rabizadeh S, Rhee KJ, Wu S, Huso D, Gan CM, Golub JE, et al. 2007. Enterotoxigenic Bacteroides fragilis: a potential instigator of colitis. Inflamm. Bowel Dis. 13: 1475-1483.

4. Toprak NU, Yagci A, Gulluoglu BM, Akin ML, Demirkalem P, Celenk T, et al. 2006. A possible role of Bacteroides fragilis enterotoxin in the aetiology of colorectal cancer. Clin. Microbiol. Infect. 12: 782-786.

5. Zou S, Fang L, Lee MH. 2018. Dysbiosis of gut microbiota in promoting the development of colorectal cancer. Gastroenterol. Rep. $(O x f)$. 6: 1-12.

6. Swidsinski A, Weber J, Loening-Baucke V, Hale LP, Lochs H. 2005. Spatial organization and composition of the mucosal flora in patients with inflammatory bowel disease. J. Clin. Microbiol. 43: 3380-3389.

7. Li S, Konstantinov SR, Smits R, Peppelenbosch MP. 2017. Bacterial biofilms in colorectal cancer initiation and progression. Trends. Mol. Med. 23: 18-30.

8. Caglar E, Kargul B, Tanboga I. 2005. Bacteriotherapy and probiotics' role on oral health. Oral Dis. 11: 131-137.

9. FAO/WHO. 2001. Joint FAO/WHO Working Group on Drafting Guidelines for the Evaluation of Probiotics in Food: Health and Nutritional Properties of Probiotics in Food Including Powder Milk with Live Lactic Acid Bacteria. Rome: Publishing Management Service, Information Division, FAO.

10. Kaur S, Sharma P, Kalia N, Singh J, Kaur S. 2018. Antibiofilm properties of the fecal probiotic Lactobacilli against Vibrio spp. Front. Cell. Infect. Microbiol. 8: 120.

11. Kemgang TS, Kapila S, Shanmugam VP, Kapila R. 2014. Cross-talk between probiotic Lactobacilli and host immune system. J. Appl. Microbiol. 117: 303-319.

12. Seki H, Shiohara M, Matsumura T, Miyagawa N, Tanaka M, Komiyama A, et al. 2003. Prevention of antibiotic-associated diarrhea in children by Clostridium butyricum MIYAIRI. Pediatr. Int. 45: 86-90.

13. Hamer HM, Jonkers D, Venema K, Vanhoutvin S, Troost FJ, Brummer RJ. 2008. Review article: the role of butyrate on colonic function. Aliment. Pharmacol. Ther. 27: 104-119.

14. Kashiwagi I, Morita R, Schichita T, Komai K, Saeki K, Matsumoto M, et al. 2015. Smad2 and Smad3 Inversely Regulate TGF-beta Autoinduction in Clostridium butyricumActivated Dendritic Cells. Immunity 43: 65-79.

15. Kuroiwa T, Kobari K, Iwanaga M. 1990. [Inhibition of enteropathogens by Clostridium butyricum MIYAIRI 588]. Kansenshogaku Zasshi 64: 257-263.
16. Takahashi M, Taguchi H, Yamaguchi H, Osaki T, Kamiya S. 2000. Studies of the effect of Clostridium butyricum on Helicobacter pylori in several test models including gnotobiotic mice. $J$. Med. Microbiol. 49: 635-642.

17. Takahashi M, Taguchi H, Yamaguchi H, Osaki T, Komatsu A, Kamiya S. 2004. The effect of probiotic treatment with Clostridium butyricum on enterohemorrhagic Escherichia coli O157:H7 infection in mice. FEMS Immunol. Med. Microbiol. 41: 219-226.

18. Hayashi A, Sato T, Kamada N, Mikami Y, Matsuoka K, Hisamatsu $\mathrm{T}$, et al. 2013. A single strain of Clostridium butyricum induces intestinal IL-10-producing macrophages to suppress acute experimental colitis in mice. Cell Host. Microbe. 13: 711-722.

19. Rhee KJ, Wu S, Wu X, Huso DL, Karim B, Franco AA, et al. 2009. Induction of persistent colitis by a human commensal, enterotoxigenic Bacteroides fragilis, in wild-type C57BL/6 mice. Infect. Immun. 77: 1708-1718.

20. Rosenblatt JE, Stewart PR. 1975. Anaerobic bag culture method. J. Clin. Microbiol. 1: 527-530.

21. Kim H, Kang SS. 2019. Antifungal activities against Candida albicans, of cell-free supernatants obtained from probiotic Pediococcus acidilactici HW01. Arch. Oral Biol. 99: 113-119.

22. Wasfi R, Abd El-Rahman OA, Zafer MM, Ashour HM. 2018. Probiotic Lactobacillus sp. inhibit growth, biofilm formation and gene expression of caries-inducing Streptococcus mutans. J. Cell. Mol. Med. 22: 1972-1983.

23. Clinical and Laboratory Standards Institute. 2011. Performance standards for antimicrobial susceptibility testing of anaerobic bacteria: Informational Supplement M11-S1. Wayne, P.A.: Clinical and Laboratory Standards Institute.

24. Jaffar N, Ishikawa Y, Mizuno K, Okinaga T, Maeda T. 2016. Mature biofilm degradation by potential probiotics: Aggregatibacter actinomycetemcomitans versus Lactobacillus spp. PLoS One. 11: e0159466.

25. Pratt LA, Kolter R. 1998. Genetic analysis of Escherichia coli biofilm formation: roles of flagella, motility, chemotaxis and type I pili. Mol. Microbiol. 30: 285-293.

26. Cady NC, McKean KA, Behnke J, Kubec R, Mosier AP, Kasper SH, et al. 2012. Inhibition of biofilm formation, quorum sensing and infection in Pseudomonas aeruginosa by natural products-inspired organosulfur compounds. PLoS One 7: e38492.

27. Pierce CG, Uppuluri P, Tristan AR, Wormley FL, Jr., Mowat E, Ramage G, et al. 2008. A simple and reproducible 96-well plate-based method for the formation of fungal biofilms and its application to antifungal susceptibility testing. Nat. Protoc. 3: $1494-1500$.

28. Fischer J, Prosenc MH, Wolff M, Hort N, Willumeit R, Feyerabend F. 2010. Interference of magnesium corrosion with tetrazolium-based cytotoxicity assays. Acta Biomater. 6: 1813-1823. 
29. Nosyk O, ter Haseborg E, Metzger U, Frimmel FH. 2008. A standardized pre-treatment method of biofilm flocs for fluorescence microscopic characterization. J. Microbiol. Methods 75: 449-456.

30. Bundgaard-Nielsen C, Baandrup UT, Nielsen LP, Sorensen S. 2019. The presence of bacteria varies between colorectal adenocarcinomas, precursor lesions and non-malignant tissue. BMC Cancer 19(1): 399.

31. Wexler HM, Tenorio E, Pumbwe L. 2009. Characteristics of Bacteroides fragilis lacking the major outer membrane protein, OmpA. Microbiology 155: 2694-2706.

32. Pumbwe L, Ueda O, Yoshimura F, Chang A, Smith RL, Wexler HM. 2006. Bacteroides fragilis BmeABC efflux systems additively confer intrinsic antimicrobial resistance. $J$. Antimicrob. Chemother. 58: 37-46.

33. Cassir N, Benamar S, La Scola B. 2016. Clostridium butyricum: from beneficial to a new emerging pathogen. Clin. Microbiol. Infect. 22: 37-45.

34. Di Martino P. 2018. Extracellular polymeric substances, a key element in understanding biofilm phenotype. AIMS Microbiol. 4: 274-288.

35. Dejea CM, Wick EC, Hechenbleikner EM, White JR, Mark Welch JL, Rossetti BJ, et al. 2014. Microbiota organization is a distinct feature of proximal colorectal cancers. Proc. Natl. Acad. Sci. USA 111: 18321-18326.

36. Jia L, Li D, Feng N, Shamoon M, Sun Z, Ding L, et al. 2017. Anti-diabetic effects of Clostridium butyricum CGMCC0313.1 through promoting the growth of gut butyrate-producing bacteria in type 2 diabetic mice. Sci. Rep. 7: 7046.

37. Chen ZF, Ai LY, Wang JL, Ren LL, Yu YN, Xu J, et al. 2015. Probiotics Clostridium butyricum and Bacillus subtilis ameliorate intestinal tumorigenesis. Future Microbiol. 10: 1433-1445.

38. Pierce JV, Bernstein HD. 2016. Genomic diversity of enterotoxigenic strains of Bacteroides fragilis. PLoS One 11: $\mathrm{e} 0158171$.

39. Nakanishi S, Tanaka M. 2010. Sequence analysis of a bacteriocinogenic plasmid of Clostridium butyricum and expression of the bacteriocin gene in Escherichia coli. Anaerobe. 16: 253-257.

40. Isono A, Katsuno T, Sato T, Nakagawa T, Kato Y, Sato N, et al. 2007. Clostridium butyricum TO-A culture supernatant downregulates TLR4 in human colonic epithelial cells. Dig. Dis. Sci. 52: 2963-2971.
41. Jia L, Shan K, Pan LL, Feng N, Lv Z, Sun Y, et al. 2017. Clostridium butyricum CGMCC0313.1 Protects against autoimmune diabetes by modulating intestinal immune homeostasis and inducing pancreatic regulatory $\mathrm{T}$ cells. Front. Immunol. 8: 1345.

42. Shang H, Sun J, Chen YQ. 2016. Clostridium Butyricum CGMCC0313.1 Modulates lipid profile, insulin resistance and colon homeostasis in obese mice. PLoS One 11: e0154373.

43. Gill PA, van Zelm MC, Muir JG, Gibson PR. 2018. Review article: short chain fatty acids as potential therapeutic agents in human gastrointestinal and inflammatory disorders. Aliment. Pharmacol. Ther. 48: 15-34.

44. Chung PY, Toh YS. 2014. Anti-biofilm agents: recent breakthrough against multi-drug resistant Staphylococcus aureus. Pathog. Dis. 70: 231-239.

45. Cordeiro RA, Aguiar ALR, Pereira VS, Pereira LMG, Portela FVM, Brilhante RSN, et al. 2019. Sodium butyrate inhibits planktonic cells and biofilms of Trichosporon spp. Microb. Pathog. 130: 219-225.

46. Seghal Kiran G, Priyadharshini S, Dobson ADW, Gnanamani E, Selvin J. 2016. Degradation intermediates of polyhydroxy butyrate inhibits phenotypic expression of virulence factors and biofilm formation in luminescent Vibrio sp. PUGSK8. NPJ Biofilms Microbiomes 2: 16002.

47. Poquet I, Saujet L, Canette A, Monot M, Mihajlovic J, Ghigo JM, et al. 2018. Clostridium difficile Biofilm: Remodeling metabolism and cell surface to build a sparse and heterogeneously aggregated architecture. Front. Microbiol. 9: 2084.

48. Pumbwe L, Skilbeck CA, Nakano V, Avila-Campos MJ, Piazza RM, Wexler HM. 2007. Bile salts enhance bacterial co-aggregation, bacterial-intestinal epithelial cell adhesion, biofilm formation and antimicrobial resistance of Bacteroides fragilis. Microb. Pathog. 43: 78-87.

49. Pumbwe L, Skilbeck CA, Wexler HM. 2008. Presence of quorum-sensing systems associated with multidrug resistance and biofilm formation in Bacteroides fragilis. Microb. Ecol. 56: 412-419.

50. Venter H, Mowla R, Ohene-Agyei T, Ma S. 2015. RND-type drug efflux pumps from Gram-negative bacteria: molecular mechanism and inhibition. Front. Microbiol. 6: 377. 